\title{
Uber den Einfluss der Leibessubstanzen der Typhusbazillen auf den Leberglykogengehalt.
}

\author{
II. Mitteillung.
}

Von

\section{Kiyosige Ogawa und Kyotaro Kikuti.}

(小川清茂・菊地 恭太郎)

(Aus dem Bakteriologischen Institut der Reichsuniversität zu Sendai, Direktor: Prof. Dr. M. Kuroya.)

(Received for publication, September 21, 1944)

\section{Einleitung.}

In Bezug auf den Einfluss der verschiedenen Leibessubstanzen der Typhusbazillen, d.h. des entfetteten Bakterienleibes, der PF, CF, des Bakterienrestes, der aus CF weiter isolierten MF, PSF und der zweischn diesen beiden letzteren liegenden $\mathrm{MF}_{4}$, auf den Leberglykogengehalt veröffentlichte einer von uns, $\mathrm{Ogaw} \mathrm{a}^{1)}$ in seiner vorigen Mitteilung, dass $\mathrm{MF}$, die am giftigsten ist, das Leberglykogen am stärksten vermindert und ziemlich rasch wiederherstellen lässt und $\mathrm{CF}$ demnächst folgt, dass PF und entfetteter Bakterienleib nur leicht das Leberglykogen vermindern, aber für seine Erholung relativ lange Zeit nötig ist, und dass Bakterienrest und PSF fast keine Einwirkung zeigen und $\mathrm{MF}_{4}$ nur bei grosser Gebrauchsmenge wie MF einwirkt.

Infolge der eingehenden Forschung von Prof. $\mathrm{Kuroya}{ }^{2)}$ und seinen Mitarbeitern kam es ans Licht, dass MF eine nicht einfach zusammengesetzte Substanz ist, sondern als Hauptbestandteile Eiweiss, Polysaccharid und Phosphatid besitzt. CF ist zum grössten Teil aus MF gebilidet, aber wahrscheinlich enthält sie ausserdem die oben genannten drei Substanzen, welche die Hauptbestandteile der MF sind, Polysaccharid (PSF) und Polypeptid, wenn auch spärlich, im freien Zustand. Um den Einfluss dieser verschiedenen Substanzen auf den Leberglykogengehalt zu beobachten und dadurch das Wesen der Einwirkung der MF klarzustellen, wurde dieses Experiment ausgeführt.

Bezüglich einer der obigen drei Substanzen, PSF ist schon in voriger Mitteilung beschrieben, hier untersuchten Verfasser die Einwirkungen von Eiweiss und Phosphatid, welche aus CF isoliert wurden, und versuchten weiter als Kontrolle den Einfluss von dem in fettiger Substanz der Typhusbazillen frei enthaltenen Phosphatid und Lecithin (Kahl- 
ba um) als Vertreter der bekannten Phosphatiden.

\section{Versuchsmaterial und -methode.}

1. Der gebrauchte Stamm, die Versuchstiere und die Glykogenbestimmungsmethode.

Sie sind ganz gleich wie in voriger Mitteilung; ihre Beschreibung ist also hier ausgelassen.

2. Isolierung der gebrauchten Leibessubstanzen.

a. Eiweiss und Polypeptid.

Durch Verfahren nach Prof. K u ro y a wurden die mit Aether-Alkohòl und Aceton entfetteten und getrockneten Bakterienleiber mit 50 facher Menge Wasser zugesetzt und in neutraler Reaktion bei $100^{\circ} \mathrm{C} 30$ Minuten lang erhitzt und dann abzentrifugiert. Auf die Niederschläge wurde die gleiche Extrahierung noch einigemal wiederholt. Die ganze gesammelte Flüssigkeit wurde bei niedriger Temperatur bis zum 1/10 ihres anfänglichen Volumens eingeengt, dialysiert und ferner im geeigneten isoelektrischen Punkt wurde die PF daraus beseitigt. Wenn man dieser Flüssigkeit in $\mathrm{pH}$ 5,0 eine 5 fache Menge Aceton zusetzte, so gewann man einen Niederschlag. d.h. CF. Nach nochmaliger Wiederholung der geleichen Behandlung wusch man den Niederschlag mit Alkohol und Aether, dann trocknete man ihn einem Exsikkator über $\mathrm{CaCl}_{2}$ und $\mathrm{P}_{2} \mathrm{O}_{5}$. Nachdem man $1 \%$ GF-Lösung mit $1 \% \mathrm{HCl}$ zu pH 2,0 brachte, mit 3 facher Menge Aceton versetzte und den Bodensatz beseitigte, dampfte man die überstehende Flüssigkeit ein und setzte bei pH 5,0 weiter eine 3 fache Menge Aceton zu. Durch diese Manipulation fällte sich eine eiweissartige Substanz. Die Biuretreaktion dieser Substanz war positiv, die Molischsche negative und 1\% Lösung derselben reagierte schwach alkalisch $(\mathrm{pH} \mathrm{8,4)}$ und zeigte keinen isoeloktrischen Punkt. Die 1\% Lösung derselben wurde mit 50\% Trichloressigsäure genügend versetzt und der dabei vorkommende Niederschlag wurde im Wasser gelöst, dialysiert und dann die in $\mathrm{pH}$ 4,8 praezipitierbare Substanz beseitigt. Aus dieser überstehenden Flüssigkeit durch Zusatz mit 3 facher Menge Aceton und mit Stückchen von Natriumacetat gewann man das Polypeptid. Diese Substanz betrug an N 9,32\%, die Molisch sche Reaktion derselben war negativ, Ninhydrin- und Biuret- positiv. Es zeigte zwischen dieser Substanz und dem Immunserum gegen Typhusbazillen eine niedrige (1:400-300) Praezipitationsreaktion. Die Giftigkeit derselben für Mäuse war so klein, dass die M.L.D. über $10 \mathrm{mg}$ betrug. Dagegen zeigte die Substanz, welche nach der Fällung durch Trichloressigsäure im isoelektrischen Punkt ( $\mathrm{pH} \mathrm{4,8)} \mathrm{isoliert} \mathrm{wurde,} \mathrm{negative} \mathrm{Molis} \mathrm{ch-}$ sche und Ninhydrinreaktion und eine positive Biuretreaktion, enthielt $15,54 \% \mathrm{~N}$ und zwischen derselben und dem Immunserum gegen Typhusbazillen zeigte sich eine ziemlich starke $(1: 4,000)$ Praezipitationsreaktion. 
Die M.L.D. für Mäuse war über $10 \mathrm{mg}$. Bei diesem Versuch brauchte man die letztere Substanz.

b. Phosphatid I.

Wenn man den oben beschriebenen Niederschlag, welcher in saurer Reaktion durch Zusatz mit Aceton vorkam, in $\mathrm{pH} 4,0$ mit wenig Wasser wiederholt spülte, so war das Wasçhwasser zuerst klar und dessen Molischsche Reaktion positiv, Biuret- negativ, aber allmählich wurde die Flüssigkeit trübe und dabei wurden die obenerwähnten beiden Reaktionen negativ. Wurde die ganze letztere Flüssigkeit mit Aceton zugesetzt, so erhielt man daraus eine phosphatidartige Substanz. Diese Substanz fällt sich durch Zusatz mit Aceton, löst sich in Aether, Alkohol und neutralem Wasser und zeigte positive Reaktion für Glyzerin durch Tüpfelanalyse. Der N-Gehalt derselben betrug $0,14 \%, \mathrm{P}-3,28 \%$ und die M.L.D. für Mäuse 0,3 mg pro $10 \mathrm{~g}$ des Körpergewichtes. Auf Grund von obenerwähnten Eigenschaften ist diese Substanz weahrscheinlich eine Art von Phosphatiden, hier provisorisch als Phosphatid I bezeichnet.

Eiweiss und Phosphatid unter den oben beschriebenen 3 Substanzen und Polysaccharid, welches schon in voriger Mitteilung beschrieben war, sind wahrscheinlich Hauptbestandteile der MF. Bei Hydrolyse der MF lassen sie sich auch in gleicher Weise herstellen. Dagegen ist es noch unklar, ob Polypeptid ein Bestandteil der Bakterienleiber ist oder nicht. Die sog. CF enthält vermultlich ausserdem MF die Spaltprodukte derselben, d.h. Phosphatid, Eiweiss, Polysaccharid und Polypeptid.

c. Phosphatid II.

Für Isolierung der Leibessubstanzen, wie bereits beschrieben, wurde die Bakterienkultur unter Zusatz zuerst mit 5 facher Menge Aceton und demnächst mit gleicher Menge Aether-Alkohol je über 10 Tage lang in Zimmertemperatur stehen gelassen und dadurch völlig entfettet. Die aus diesem Extrakt durch Abdampfung unter der Leitung der Kohlensäure bei niedriger Temperatur gewonnene Substanz wurde mit Aether versetzt. Wenn man der letzteren Aetherlösung eine 2 fache Menge Aceton zusetzte, so kam ein Niederschlag vor. Wenn man diese Substanz im Kohlensäuregas trocknete, so bekam man eine Art von Phosphatiden. Dieses Phosphatid wurde durch wiederholte Lösung in Aether und Fällung mit Aceton gereinigt. Diese Substanz ist sehr hygroskopisch und im Wasser leicht emulsierbar. Der N-Gehalt derselben betrug 1,95\%, P- 3,68\% und die Glyzerinreaktion war positiv. Die obigen Eigenschaften stimmen mit denen von Lecithin oder Kephalin gut überein. Die Giftigkeit derselben für Mäuse war so klein, dass die mit $10 \mathrm{mg}$ pro $10 \mathrm{~g}$ Körpergewicht injizierten Tiere fast keine Symptome zeigten. Hier bezeichnet man provisorisch diese Substanz als Phosphatid II. 


\section{Versuchsergebnisse.}

A. Kontrollversuch.

Weil dieses Experiment zwischen September und November durchgeführt wurde, wurde als Kontrolle der Leberglykogengehalt der normalen Mäuse im Oktober gemessen. Der Wert betrug 1,61-2,60\%, durchschnittlich 2,12\%. Der Versuch mit Lecithin wurde jedoch aus gewissen Gründen im August vorgenommen, daher wurde auch der Normalwert des Leberglykogens in der betreffenden Jahreszeit bestimmt. Der Wert betrug $0,91-1,89 \%$, durchschnittlich 1,36\%. Diese Tatsache steht wahrscheinlich in Beziehung dazu, dass der Widerstand der Mäuse für Typhusoder Dysenteriebazillen sich im Sommer vermindert, worüber Verfasser z.Zt. weiterhin Studien macht.

Der Einfluss durch die Einführung der physiologischen Kochsalzlösung und durch den Hunger wurde schon in der vorigen Mitteilung besprochen.

B. Versuche mit Leibessubstanzen.

1. Glykogengehalt nach der Injektion von dem aus $\mathrm{MF}$ isolierten Eiweiss (MPF). Bei Injektion von 5,0 $\mathrm{mg}$ pro $10 \mathrm{~g} \mathrm{Körpergewicht.}$

Nur 1 Stunde nach der Injektion vermindert sich das Leberglykogen bis zu $0,39-0,73 \%$, durchschnittlich $0,55 \%$, demnach bis 24 . Stunde bleibt es bei $0,5 \%$. Nach 48 Stunden vermehrt es sich etwas und nach 72 Stunden erreicht es bei einigen Fällen den Normalwert, aber der durchschnittliche Wert desselben beträgt noch 1,32\%.

Bei Injektion von 1,0 mg pro $10 \mathrm{~g}$ Körpergewicht.

Eine Stunde nach der Injektion vermindert sich das Leberglykogen bis durchschnittlich 1,24\%, nach 3 Stunden bis $0,9 \%$ und danach bis zur 24. Sunde bleibt es bei ungefähr $0,9 \%$. Nach 48 Stunden nimmt es bis $1,3 \% \mathrm{zu}$, nach 72 Stunden bis $1,55 \%$, aber es ist noch niedriger als der Normalwert.

Bei Injektion von $0,2 \mathrm{mg}$ pro $10 \mathrm{~g}$ Körpergewicht.

Die Abnahme des Leberglykogens ist sehr leicht. Binnen 24 Stunden neigt es zu leichter Verminderung, bleibt aber immer über 1,0\%, ausgenommen von einigen Fällen.

Ohne Rücksicht auf den Unterschied der Gebrauchsmenge ist der Verlauf nach der MPF-Injektion sehr ähnlich dem Fall von PF in voriger Mitteilung. Die zu dem Eiweisssystem gehörigen Substanzen, wie Bakterienleiber selbst, PF oder MPF, haben auf diese Weise folgende Eigenschaften: Die Leberglykogen vermindernde Fähigkeit ist schwach, aber die Einwirkung dauert verhältnismässig lang.

2. Glykogengehalt nach der Injektion von phosphatid I.

Bei Injektion von $0,1 \mathrm{mg}$ pro $10 \mathrm{~g}$ Körpergewicht. 
Das Leberglykogen vermindert sich rasch und intensiv, wie nach 1 Stunde $0,71 \%$, nach 3 Stunden $0,27 \%$, nach 6 Stunden $0,20 \%$, und nach 12 Stunden ereicht es einen minimalen Wert, durchschnittlich 0,19\%; bei einem Fall zeigt sich sogar kein Glykogen. Danach wendet es sich nach Wiederherstellung zu und erreicht nach 48 Stunden ganz den Normalwert.

Bei Injektion von $0,02 \mathrm{mg}$ pro $10 \mathrm{~g} \mathrm{~K}$ örpergewicht.

Auch bei diesem Fall nimmt das Leberglykogen relativ auffallend ab, wie nach 1 Stunde $1,02 \%$, nach 3 Stunden $0,75 \%$, nach 6 Stunden $0,61 \%$ und nach 12 Stunden 0,59\%, aber von 12. Stunde an nimmt es den umgekehrten Verlauf und nach 24 Stunden beträgt es etwas den Normalwert übertreffend durchschnittlich $2,40 \%$.

Bei Injektion von $0,005 \mathrm{mg}, 10 \mathrm{~g} \mathrm{Körpergewicht.}$

Nach 3 Stunden vermindert sich das Leberglykogen bis zu 0,87\%, aber danach kehrt es so rasch zurück, dass es nach 6 Stunden 1,50\% zeigt und nach 12 Stunden fast den normalen Zustand erreicht.

Im Vergleich mit den Fällen von MFP ist die Glykogenabnahme nach der Injektion von Phosphatid I stärker, dagegen die Dauer derselben kürzer. Inşbesondere ist erwähnenswert, dass die Kurve bei der Injektion von $0,005 \mathrm{mg}$ Phosphatid I ganz ähnlich wie die bei MF in voriger Mitteilung verläuft.

3. Glykogengehalt nach der Injektion von Phosphatid II.

Bei Injektion von 5,0 mg pro $10 \mathrm{~g}$ Körpergewicht.

In 1 . Stunde vermindert sich das Leberglykogen bis auf $0,54 \%$ und danach bis zur 24. Stunde bleibt es ungefähr bei $0,5 \%$. Nach 48 Stunden wird es bis zur Hälfte des Normalwertes, d.h. 1,04\%, wiederhergestellt.

Bei Injektion von 1,0 mg pro $10 \mathrm{~g}$ Körpergewicht.

Innerhalb der ersten 12 Stunden bleibt das Leberglykogen bei 1,0\% und nach 24 Stunden wird es bis zu 1,36\% wiederhergestellt.

Bei Injektion von $0,2 \mathrm{mg}$ pro $10 \mathrm{~g}$ Körpergewicht.

Bei diesem Fall ist die Abnahme des Leberglykogens viel leichter. Nach 1 Stunde kommt es zum minimalen Wert $(1,35 \%)$ und danach wendet es sich so schnell zur Erholung, dass es nach 12 Stunden schon 1,68\% erreicht.

Bei der Injektion von Phosphatid II ist die Abnahme des Leberglykogens leicht, aber für die Wiederherstellung desselben scheint es relativ längerer Zeit zu bedürfen.

4. Glykogengehalt nach der Injektion von Lecithin.

Dass das Leberglykogen durch Einführung von Lecithinemulsion sich vermindert ist von Cruto-Alfonso, ${ }^{3)} \mathrm{Francesc0^{4 }}$ und Otomo und $\mathrm{Nag} \mathrm{a} \mathrm{o}^{5}$ u.a. schon angegeben. Um die Ergebnisse durch die oben beschriebenen 2 Phosphatide, welche aus Typhusbazillen isoliert waren, zu 
vergleichen, injizierte man den Mäusen intraperitoneal mit verschiedenen Mengen von Lecithin und gewann folgende Resultate.

Bei Injektion von 5,0 mg pro $10 \mathrm{~g} \mathrm{Körpergewicht.}$

Nach 1 Stunde zeigt das Leberglykogen $0,66 \%$, danach vermindert es sich allmählich und erreicht nach 12 Stunden den minimalen Wert, $0: 3 \%$. Daraufhin wendet es sich wieder allmählich zur Erholung und zeigt nach 48 Stunden völlige Wiederherstellung.

Bei Injektion von 1,0 mg pro $10 \mathrm{~g} \mathrm{Körpergewicht.}$

Die Abnahme des Leberglykogens ist so leicht, dass es nach 12 Stunden zu einem minimalen Gehalt von 0,81\% kommt und nach 24 Stunden völlig wiederhergestellt wird.

Bei Injektion von $0,2 \mathrm{mg}$ pro $10 \mathrm{~g}$ Körpergewicht.

Die Abnahme des Leberglykogens ist viel leichter, bleibt immer über $1 \%$ und fast kein bemerkenswerter Einfluss ist nachweisbar.

\section{Zusammenfassung und Besprechung.}

Obwohl das aus MF isolierte Eiweiss MPF im Vergleich mit PF weniger giftig und daher zwar eine grössere Dose nötig ist, eine fast gleiche Verminderung des Leberglykogens hervorzurufen, verläuft die Schwankung des Leberglykogengehaltes ganz ähnlich, wie die bei PFEinführung in voriger Mitteilung. Überdies ist es sehr merkwürdig, dass Phosphatid I, welches auf Mäuse stark giftig wirkt, auch das Leberglykogen stark vermindert, aber diese Beeinflussung sehr schnell verliert und besonders die Abnahme bei Einführung von $0,005 \mathrm{mg}$ einen ganz ähnlichen Verlauf wie bei MF in voriger Mitteilung nimmt. Unter den 3 Hauptbestandteilen der MF ist MPF kaum giftig und deren Einwirkung auf Leberglykogen schwach, Polysaccharid, wie in voriger Mitteilung beschrieben, fast weder giftig noch von Leberglykogen vermindernder Fähigkeit und nur Phosphatid ist giftig, und dadurch wird eine starke Einwirkung auf Leberglykogen bewiesen. Aus den obigen Punkten wird die Einwirkung der MF damit erklärt, dass Phosphatid in MF, d.h. Phosphatid I bei diesem Versuch, eine Hauptrolle spielt und Eiweiss und Polysaccharid daran teilnehmen. Diese Ansicht wird auch durch die Arbeit von Prof. Kuroya ${ }^{6)}$ und seinem Mitarbeiter bestätigt. Sie bemerkten in einer Veröffentlichung über die Beziehung zwischen der Giftigkeit und chemischen Zusammensetzung der verschiedenen MF, dass, je giftiger die MF ist, desto mehr $\mathrm{N}$ und $\mathrm{P}$ in derselben enthalten sind und diese $\mathrm{N}$ und $\mathrm{P}$ haltigen Substanzen mit Polysacchrid fest verbunden sind. Die Leberglykogen vermindernde Fähigkeit von Phosphatid II und Lecithin ist fast gleichgradig und im Vergleich mit Phosphatid I viel schwächer. Die Ergebnisse bei gleicher Gebrauchsmenge, d.h. 0,2 mg (bei Phosphatid I 0,1 mg), zeigen; wie in Fig. 1 ersichtlich ist, deutlichen Unterschied zwischen diesen 3 
Fig. 1. Vergleich der Einflüsse durch die Einführung von Phosphatid I, II und Lecithin.

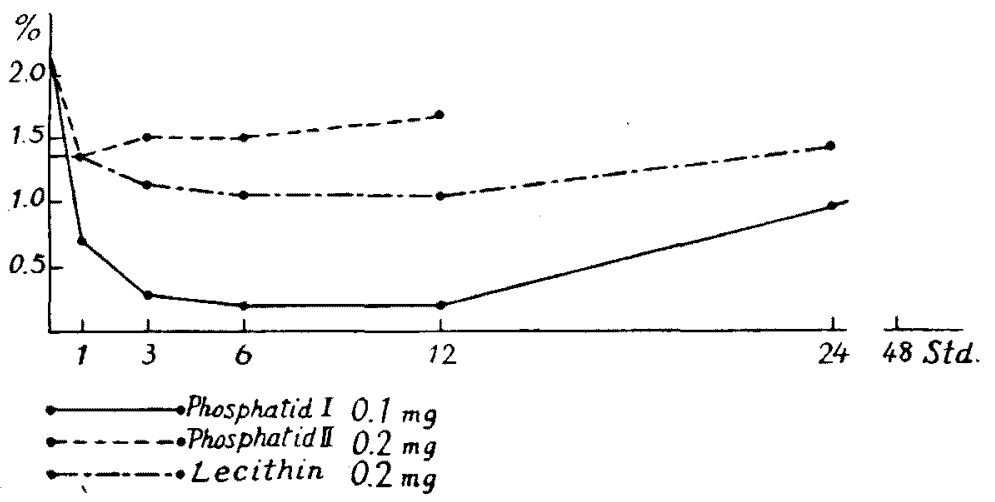

Phosphatiden. Nämlich Phosphatid I, welches ein Bestandteil der MF ist, übt einen besonders starken Einfluss aus, welcher das letztere von freiem Phosphatid II oder Lecithin biologisch deutlich unterscheiden lässt.

Fig. 2. Vergleich der Einflüsse durch die Einführung der CF, MF und des Phosphatid I in gleicher Menge $(0,02 \mathrm{mg})$.

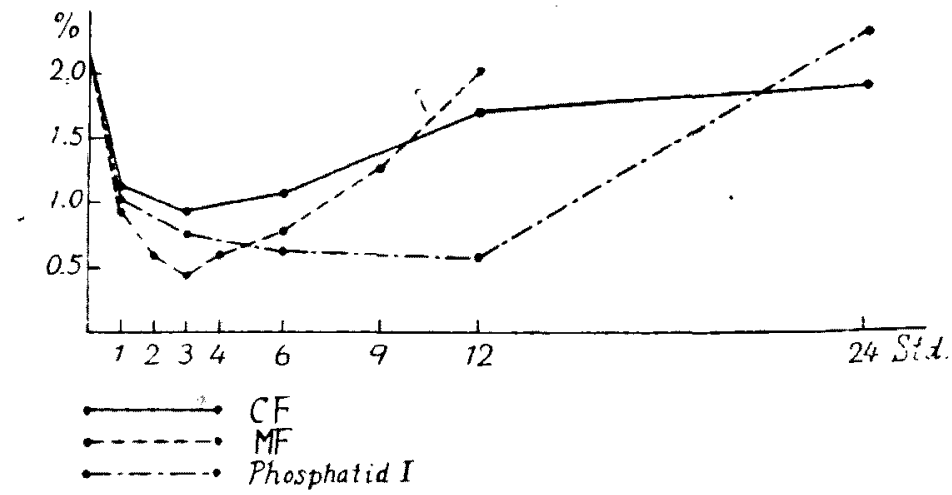

Vergleicht man nun die Einflüsse durch je $0,02 \mathrm{mg}$ von $\mathrm{CF}$, MF und Phosphatid I, wie in Fig. 2 ersichtlich ist, so zeigen alle diese Substanzen eine deutliche Leberglykogen vermindernde Wirkung. Dies ist daraus leicht zu verstehen, dass der Hauptbestandteil der CF die MF ist und die Wirkung der MF wesentlich dieselbe des Phosphatides.

\section{Schluss.}

Die Ergebnisse der Versuche über die Einwirkungen der Leibessubsstanzen der Typhusbazillen auf den Leberglykogengehalt, worüber in voriger und dieser Mitteilung berichtet ist, werden folgenderweise zusammengefasst:-

1) Die hauptsächlich von Eiweiss und seinem Spaltungsprodukte 
zusammengesetzten Leibessubstanzen, wie entfettete Bakterienleiber, PF und MPF, bedürfen einer grösseren Menge um das Leberglykogen deutlich zu vermindern, und dabei dauern die Einwirkung verhältnismässig länger. Unter diesen 3 Substanzen wirkt die PF am stärksten.

2) Dagegen zeigen CF und MF, welche einer giftigen Polysaccharidgruppe angehören, und Phosphatid I, welches für den Hauptbestandteil der beiden letzteren zu halten ist, alle in kleiner Gebrauchsmenge eine bedeutende Leberglykogen vermindernde Fähigkeit. Diese Einwirkung scheint im allgemeinen sehr rasch vor sich zu gehen und nach kurzer Dauer abzulaufen. Under diesen 3 Substanzen zeigt MF die stärkste Wirkung.

3) Bei allen obenerwähnten Substanzen steht im allgemeinen die Leberglykogen vermindernde Fähigkeit im geraden Verhältnis zur Giftigkeit und bei derselben Substanz zur Gebrauchsmenge.

4) Phosphatid II, das im freien Lipin des Typhusbazillen enthalten ist, zeigt eine schwache, Leberglykogen vermindernde. Wirkung; dagegen Phosphatid I, das aus giftiger Leibessubstanz isoliert ist, eine starke Wirkung.

\section{Literatur.}

(1) Ogawa, Tohoku J. Exp. Med., 1948, 49, 365.

(2) Ku roya, Dai 16 kai Rengo-Biseibutugakkai Kiroku, 1943, 310.

(3) Cruto u, Alfonso, zit. n. Kongresszentrabl. f. ges. inn. Med., 1910, 55, 209.

(4) Francesco, zit. n. Kongresszentrabl.f. ges. inn. Med., 1933, 70, 387.

(5) Otomou. Nagao, Tohoku J. Exp. Med., 1935, 25, 63.

(6) Kuroya u. Koizumi, Tohoku Igaku Zassi, 1941, 29, 231. 